\title{
L'ethnologie face aux sociétés rurales *
}

Entre toute autre chose, c'est peut-être l'étude de la "différence» qui permet de comprendre la vocation fondamentale de la discipline que nous appelons Ethnologie. Aujourd'hui, cette vocation naît d'une situation historique nouvelle qui voit l'occident industrialisé conquérir progressivement tous les espaces «autres» (c'està-dire, toute réalité sociale se représentant différente de ce modèle) et ces espaces y résister (ou non) de différentes façons.

La spécificité de cette ethnologie ne se situe donc pas par rapport à des «objets» particuliers mais en fonction d'une problématique.

En ce qui concerne le sujet présentement traité, les sociétés rurales, cette problématique est définie par un regard qui tient compte à la fois de la dimension historique et du contenu globalisant des faits sociaux. Dans ce sens, on pourrait peut-être prétendre qu'une «ethnologie rurale» n'existe pas! Ce qui existe, c'est un regard ethnologique sur les sociétés rurales, dans la phase actuelle de leur histoire.

Cet article se propose de discuter:

- tout d'abord du concept de société rurale par rapport à celui, dominant, de paysannerie;

- de montrer ensuite quelques éléments de la démarche proposée par/à l'ethnologue en vue d'interpréter la complexité du monde rural;

- pour conclure, nous présenterons quelques réflexions sur les conséquences (surtout d'ordre éthique) que nous considérons comme découlant de cette démarche.

\section{Le flou de certains concepts}

Force nous est de constater qu'une grande confusion règne dans le domaine des terminologies quand il s'agit de préciser des mots et expressions comme paysans, paysannerie, sociétés paysannes, sociétés agricoles, agriculteurs, monde agricole, etc...et il n'existe aucune définition dont l'arbitrage viendrait clarifier le débat.

Devant une telle situation, notre propos n'est pas de vouloir tout éclaircir en ignorant le danger de tout compliquer. Néanmoins nous nous permettrons de

* Les idées traitées dans cet article ont fait l'objet de discussions de l'Atelier de Recherche Anthropologique (Lausanne). dire quelques mots sur les relations entre concept de paysannerie et concept de société rurale.

\section{La paysannerie}

Remarquons qu'on parle de paysannerie et non pas de "société», de catégorie ou d'une classe; il s'agit d'une "couche» de population, partie intégrante d'une unité plus vaste qui est l'Etat-nation.

Selon les différentes tendances, cette couche se situe à mi-chemin entre l'urbain et le "sauvage» (Redfield, Mendras,...) ou elle constitue un des pôles des relations économiques d'exploitation engendrées par le capitalisme (Jollivet,...). Chez les ruralistes, la vision qu'ils portent mélange évolutionnisme et misérabilisme. Pour les uns, la paysannerie est composée d'êtres à moderniser, voire à technifier, pour qu'ils entrent de plein droit et totalement dans les rouages du système marchand; pour les autres, ils sont des alliés difficiles et douteux de la classe ouvrière qu'on se doit de regarder toujours avec méfiance. $\mathrm{Ni}$ pour les premiers, ni pour les seconds, le monde paysan s'insère d'une manière déterminante dans l'histoire d'aujourd'hui. Quant à la question de l'existence d'une société rurale ayant une spécificité propre, se reproduisant selon des rapports sociaux de nature particulière... cette question n'est pas posée de façon claire. Nous pensons que les insuffisances qui perpétuent ce type de situation sont de trois ordres:

1. une connaissance imparfaite de la logique qui domine la société industrielle et qui, en même temps, l'unifie;

2. une absence totale d'éclairage provenant des sociétés rurales dites "primitives» ou "sauvages» situées, en général, en dehors de l'emprise directe du système industriel;

3. une dominance de l'approche praxéologique qui conditionne l'analyse (et en dernière instance la connaissance) à sa finalité, celle de la manipulation transformation. Nous nous référons essentiellement aux différentes théories de la modernisation, du changement social.

Comment éviter de tomber, d'une part, dans la réduction matérialiste et productiviste qui domine aujour-

Dr. Didier Delaleu, Institut d'Ethnologie, Neuchâtel et Dr. Fabrizio Sabelli, Atelier de Recherche Anthropologique, Lausanne. 
d'hui parmi les sociologues marxistes et non-marxistes? Comment éviter, d'autre part, de nous enfermer dans une vision étroitement "ethnologique» qui consisterait à n'explorer que le rural «primitif» ou «sauvage»? C'est à ce problème que nous allons essayer de répondre en abordant le concept de société rurale.

\section{Des sociétés rurales}

L'éclairage ethnologique doit porter sur les sociétés rurales en général, à l'intérieur desquelles les paysans peuvent «se retrouven». Le concept de société rurale repose sur l'idée de sociabilité.

Ce point nous amène à envisager la notion de ruralité comme un ensemble de rapports sociaux spécifiques (économiques, des pratiques sociales,...) qui se créent et se reproduisent dans les campagnes, du fait des habitants de ces campagnes, lesquels se pensent comme "groupe» en référence à ces rapports spécifiques. En d'autres termes, UNE ruralité est la traduction d'un niveau de pratiques endogènes qui génèrent un mode de re-production particulier, pour un groupe unique, dans un milieu rural spécifique.

Deux idées centrales sont ici sous-jacentes qui induiront une appréhension plus fine des sociétés rurales:

1. identité fondée sur une spécificité des rapports sociaux et des systèmes internes de communication; 2. conditions de re-production de cette spécificité.

L'identité et la re-production nous amènent à parler de l'autonomie, condition essentielle de leur existence. De quelle manière intervient l'idée d'autonomie dans la définition des différents concepts?

«Une société paysanne est un ensemble relativement autonome au sein d'une société globale plus large. Si une société agraire est complétement autonome et ne fait pas fonctionellement partie d'une société plus large, nous parlerons de société "sauvage», au sens que le XVIIIème siècle donnait à ce mot et qui paraît plus adéquat que les termes actuels (primitif, archaique, sans écriture, sans histoire, etc...). Si les collectivités rurales ne jouissent pas d'une relative autonomie par rapport à la société englobante, nous parlerons d'agriculteurs, de groupes locaux, éventuellement de «classes» rurales, mais non de paysannerie. En un mot, le paysan se définit par rapport à la ville. S'il n'y a pas de ville, il n'y a pas de paysan, et si la société entière est urbanisée, il n'y en a pas non plus.» (MENDRAS, 1970: 14).

L'ethnologue ne mélange pas le fait de vivre à la campagne et la ruralité. Ceci implique qu'une des difficultés de la recherche en ethnologie rurale européenne est de savoir différencier les rapports de production et de sociabilité de type industriel de ceux qui répondent à la définition de la ruralité que nous avions auparavant donnée; de savoir les confronter à un ensemble de situations historiques qui gardent ces deux «logiques» et qui évoluent dans un sens ou dans un autre, en fonction du pouvoir de domination ou du pouvoir de résistance.
L'ethnologie ne saisit pas uniquement les sociétés rurales en fonction de la dépendance économique mais aussi et surtout en fonction du mode d'existence.

Dans un sens tout à fait différent, $M$. Jollivet conçoit les sociétés rurales uniquement en fonction de l'évolution de l'industrie:

«C'est donc une théorie de la place des «sociétés» rurales dans le développement du mode de production capitaliste qu'il convient de faire. Or ces «sociétés» rurales et leur évolution sont historiquement liées à la formation et aux transformations de la paysannerie. S'interroger sur les «sociétés» rurales, c'est avant tout s'interroger sur le devenir de la paysannerie dans le mode de production capitaliste et plus précisément, sur l'évolution que connaissent et la fonction que jouent les cadres sociaux de la vie rurale dans ce devenir et par rapport à ce devenir.» (JOLLIVET, 1974: 230). Ce n'est pas obligatoirement faire œuvre de passéiste que de vouloir remettre en cause certaines analyses de ce type si nous cherchons la raison d'un tel aboutissement dans les insuffisances que nous relevions précédemment. S'il est vrai que «les campagnes sont en train de cesser d'être avant tout l'espace agricole et l'espace des "sociétés paysannes», pour être progressiment gagnées par l'industrialisation»" (JOLLIVET, 1974: 230), cette constatation (car il ne s'agit pas ici d'analyse) ne nous dit rien du comportement spécifique des sociétés rurales face à ce processus présenté comme inéluctable.

Ce n'est pas obligatoirement être romantique que de vouloir porter un regard nuancé sur la différence pour analyser les réponses spécifiques que fournissent ces sociétés à «une théorie de l'espace «rural»et du peuplement «rural» comme simple forme d'habitat» (JOLLIVET, 1974: 230), à un système englobant et uniformisant.

$\mathrm{Ne}$ pas croire à l'évolutionnisme unilinéaire, quand bien même il s'appliquerait au développement de ce système uniformisant, ne peut être jugé de passéisme même si, dans bien des cas, l'optimisme est nécessaire.

\section{La complexité du rural et l'éclectisme de l'ethnologie}

Contrairement à certaines positions de l'ethnologie «traditionnelle» qui divise les groupements humains en sociétés «simples» (rurales, primitives, sauvages, etc...) et sociétés "complexes» (urbaines, industrielles, modernes, etc...), l'ethnologie découvre dans les sociétés rurales un lieu de complexité extrême quand il s'agit de saisir la sociabilité sous toutes ses facettes et son imbrication dans tous les niveaux de la vie collective, notamment dans l'organisation productive. La démarche ne peut être conçue que comme pluridisciplinaire (dans la mesure où des disciplines «fixes» existent). Ainsi la lecture ethnologique des sociétés 
rurales ne se fera que par la mise en œuvre de plusieurs "savoirs» (agronomie, histoire, écologie, économie, droit, etc...) dans une unité de regard, et dont l'imbrication aboutira à une vision globalisante de la situation historique des groupes choisis (ou se désignant) dans la recherche.

Cette démarche qui consiste à saisir le haut degré de complexité et de différenciation de ces sociétés empêche (ou devrait empêcher) de tomber dans les pièges du réductionnisme, erreur typique aux études économiques ou sociologiques. En effet, on a trop souvent tendance à considérer les sociétés rurales comme de simples «objets» de l'histoire, d'une histoire qui évolue en fonction des étapes suivies et des pas franchis par le système industriel au cours de son développement. L'éclairage ethnologique devrait mettre en relief la créativité sociale, les facultés d'adaptation face au milieu, l'imagination collective qui se développent à l'occasion des conflits; cet éclairage ethnologique ferait ainsi ressortir les sociétés rurales comme de véritables agents historiques malgré le fait qu'une partie plus ou moins importante de «leun histoire échappe à leur contrôle.

Le processus d'uniformisation générale inscrit dans celui du développement industriel se trouve ainsi confronté à des obstacles parfois irréductibles; ceux-ci sont construits par des collectivités ayant accumulé un savoir écologique qui se concilie mal avec ce projet de transformation uniformisante de toute réalité matérielle.

On comprend ainsi pourquoi l'ethnologie retrouve son terrain privilégié dans l'étude des sociétés vivant en relation avec un écosystème fragile ou rude. C'est pourquoi il nous semble que les sociétés rurales ne subsistent actuellement que dans des zones périphériques où la productivité économique, conçue selon les critères de la rentabilité marchande, est très faible.

Dans de nombreux cas, leur degré d'autonomie laisse en grande partie inaltéré leur pouvoir de reproduction, fonction de la spécificité du milieu et de l'expérience historique.

Par l'utilisation de nombreux instruments empruntés aux autres disciplines, par un regard spécifique qui donne une cohérence à cette utilisation, la pratique ethnologique cherche ainsi à interpréter ce mode de reproduction qu'on pourrait appeler le «projet social» de chaque groupe concerné.

Les composantes du projet social devraient se repérer par une connaissance, même en surface, des liaisons entre passé et présent. En ce sens, «projet» ne signifie pas uniquement "projection vers le futun", mais également «trajectoire» par insertion totale dans le présent. En effet, les nombreuses recherches ethnologiques sur les sociétés rurales montrent que les populations en question n'anticipent pas le temps dans le but de maîtriser l'avenir. Leurs organisations sociales se fondent essentiellement sur une accumulation d'expériences (scientifiques) que l'on appelle parfois, avec une connotation méprisante, la «tradition». Ces organi- sations sociales se fondent également sur une créativité du présent qui fournit à ces populations le moyen de répondre aux contraintes provenant de l'extérieur (nature, politique, etc...).

Le projet social serait la concrétisation historique du savoir social, ensemble des pratiques vivantes (en partie institutionnalisées et en partie spontanées). Le projet social plonge ses racines dans l'histoire profonde du groupe, traverse les générations et entre parfois en conflit avec des projets allogènes, étrangers au groupe. 1) Le projet social, "concrétisation historique du savoir social», suppose bien évidemment un «espace» où peut se déployer l'ensemble de ses pratiques et où se grave l'histoire de leur spécificité: Le Territoire!

Mais: «Dire d'une civilisation qu'elle est aussi un Territoire, que des arbres, des fleurs (une partie ou une «façon» de l'existence des arbres ou des fleurs) en font partie, en sont éléments, tout comme il en est des hommes, ne signifie point qu'une civilisation soit attachée, liée à un territoire donné et immuable. Sédentaire ou nomade, une civilisation est une alliance entre chacune de ses parties, hommes et terre. Quelle que soit la façon de cette alliance, il n'y a civilisation qu'à sa condition, et cette condition est précisément la donnée d'un territoire, d'une existence. L'alliance peut figer, dans une sorte de temps long, les hommes et un lieu donné, elle peut aussi se faire dans le cadre de déplacement continuel, s'élaborer à l'aide de lieux toujours éphémères, n'importe, la «territorialité» (laquelle n'est qu'une partie de la cosmicité) est présente; paysans sédentaires ou nomades, citadins sédentaires ou nomades, éleveurs sédentaires ou nomades, ou encore le mélange de ces diverses formules, tous savent la Terre, le cosmos, pour peu qu'ils se définissent une identité, que se définisse une civilisation» (JAULIN, 1977: 33-34). 2)

Max Sorre prétendait: «La région, c'est l'aire d'extension d'un paysage»; sans nous prononcer sur le bienfondé d'une telle définition, nous dirons, pour notre part, que le Territoire est l'aire d'extension des «relations quotidiennes d'existence» spécifiques à un groupe (il est à noter, en outre, que l'alliance hommes-Terre évoquée par Jaulin oblige à de nouvelles interrogations du possibilisme de Vidal de la Blache).

Pour en revenir à notre propos particulier, les phénomènes de désagrégation de la société rurale s'expliquent en grande partie par la «victoire» de projets allogènes sur les projets sociaux propres aux groupes, aux collectivités rurales; et bien souvent victoire, justement par des stratégies de «déterritorialisation» physique ou visant à supprimer tout «possibilisme (remembrements parcellaires, etc... pour les sociétés rurales; frontières politiques, etc... pour les pasteurs nomades;...).

1) Pour plus de détails sur le projet social, voir DELALEU \& SABELLI, 1978: 18-39.

2) Nous ne faisons pas ici de différence entre ce que Jaulin nomme civilisation et ce que nous réunissons sous "projet social» et "groupe concerné». 
Il est évident qu'en privilégiant l'étude de la reproduction globale des systèmes sociaux, l'ethnologie ne se cantonne pas dans la recherche de l'exotique ou de l'éphémère. Le souci de dévoiler les conflits entre l'urbain et le rural, de comprendre les modalités de domination des centres industriels vis-à-vis des communautés rurales, est toujours présent; ce sont ces préoccupations qui justifie une ethnologie comme nous l'envisagions dans l'introduction: comme pratique sociale historique.

On oserait même affirmer que l'ethnologie s'acquitte de cette tâche d'une manière plus satisfaisante que beaucoup d'autres disciplines car son approche lui permet de comparer des situations historiques différenciées, sans tomber dans le piège d'une vision évolutionniste rigide.

\section{L'engagement de l'ethnologue}

Revenons aux propos généraux émis dans l'introduction. Nous espérons avoir été suffisamment clairs: la discussion sur les sociétés rurales nous a permis d'ébaucher ce que devrait être un contenu de la pratique ethnologique et, par-là même, de repérer les grandes lignes de ce qui constitue, à notre avis, sa spécificité dans l'étude de la différence.

Un point est resté en suspens: celui des conséquences d'une telle pratique sur le plan de l'engagement du chercheur.

Il nous semble évident (puisqu'il ne s'agit pas d'une science) que l'ethnologie ne doive pas se soumettre nécessairement à l'impératif de la neutralité. L'ethnologue étudie, "connaît», interprète, traduit, intervient parfois, sans pour autant concevoir cet ensemble d'activités comme répondant à des canons établis a priori par un statut figé. On peut ainsi parler d'une discipline "ouverte» mais en même temps contraignante sur le plan de l'éthique.

Le sort réservé aux sociétés rurales par l'évolution actuelle du système industriel constitue la toile de fond à tout effort d'analyse émettant des prétentions cognitives.

Le savoir ethnologique est forcément un savoir historique, et le questionnement des sociétés rurales débouche nécessairement sur une interrogation de fond sur nous-même. Cette interpellation de l'ethnologue par les sujets de sa recherche renverse paradoxalement la démarche «classique» et confie à la discipline les prémisses pour un renouveau de son statut et de ses méthodes.

\section{Bibliographie des ouvrages cités}

DELALEU, D. et F. SABELLI, 1978: L'enquête-sondage. Méthode et problèmes. Institut d'Ethnologie, Neuchâtel.

JAULIN, R., 1977: Les chemins du vide. Paris: Christian Bourgeois.

JOLLIVET, M., 1974: «Sociétés rurales et capitalisme» in Les collectivités rurales françaises T. II, pp. 230-265: Paris: A. Colin.

MENDRAS, H., 1970: La fin des paysans. Paris: A. Colin.
Fotos aus dem Fachbuch Länderkunde "JAPAN" Hans Boesch †. 256 Seiten mit 29 Kartenskizzen und 42 Farbbildern. Format $17 \times 24 \mathrm{~cm}$. Fr. 48.(C) Kümmerly + Frey, Geographischer Verlag, Bern. 

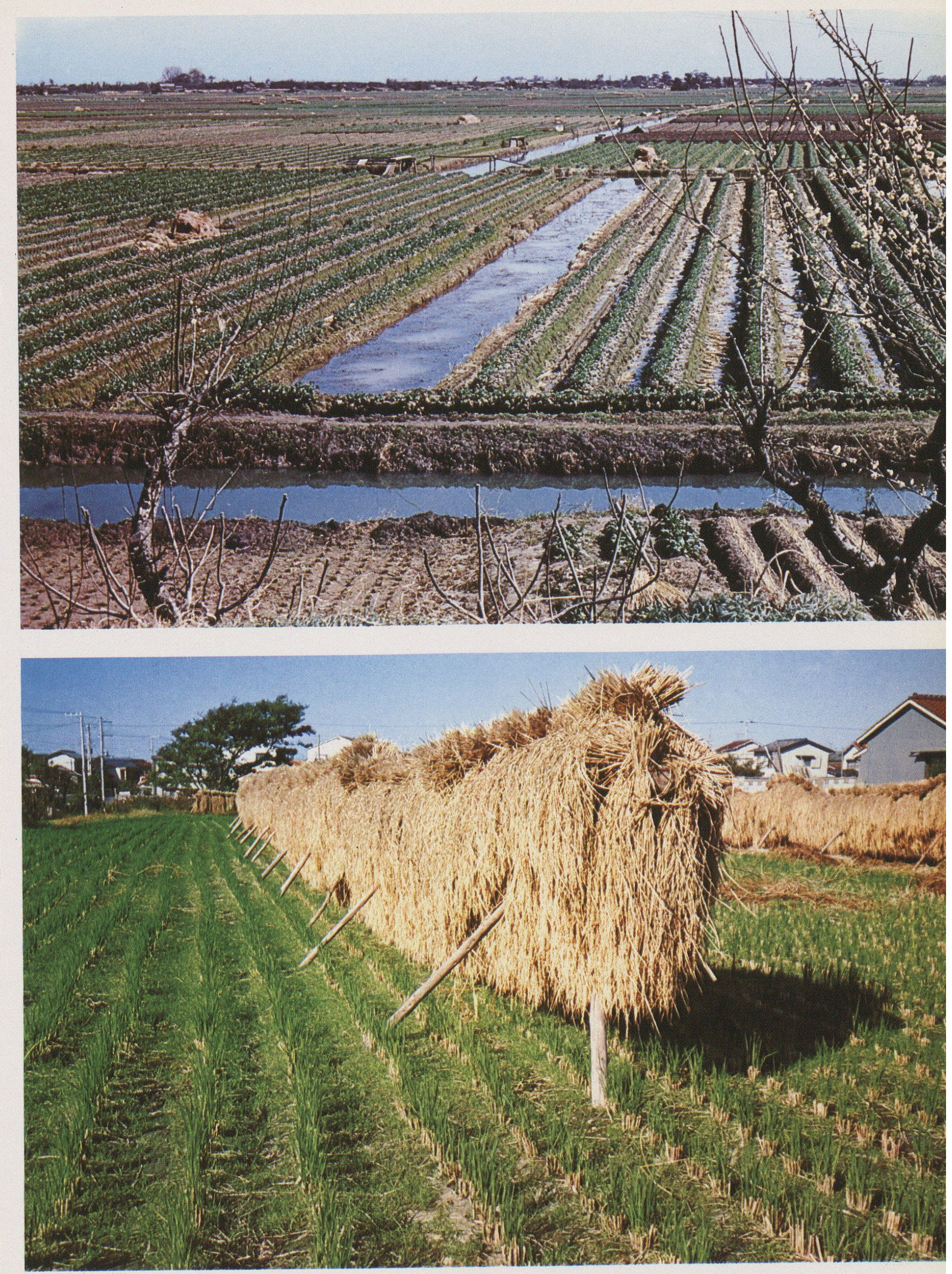

Oben Küstenebene unweit Nagoya (Aichi) im Frühling. Die Reisfelder werden noch von Winterkulturen eingenommen. Unten Herbst: gebündelter Reis, zum Austrocknen aufgehängt. Harima-Küste westlich von Akashi (Hyogo). 